\title{
A Geometrical Approach of Quasiperiodic Tilings
}

\author{
Christophe Oguey, Michel Duneau, and André Katz \\ Centre de Physique Théorique, Ecole polytechnique, F-91128 Palaiseau Cedex, France
}

\begin{abstract}
Tilings provide generalized frames of coordinates and as such they are used in different areas of physics. The aim of the present paper is to present a unified and systematic description of a class of tilings which have appeared in contexts as disconnected as crystallography and dynamical systems. The tilings of this class show periodic or quasiperiodic ordering and the tiles are related to the unit cube through affine transformations. We present a section procedure generating canonical quasiperiodic tilings and we prove that true tilings are indeed obtained. Moreover, the procedure provides a direct and simple characterization of quasiperiodicity which is suitable for tilings but which does not refer to Fourier transform.
\end{abstract}

\section{Introduction}

The present paper is devoted to the construction and analysis of two kinds of tilings of the euclidean space $\mathbf{R}^{n}$ : 1 . "Oblique" periodic tilings. 2. "Canonical" quasiperiodic tilings, as we may call them.

The tilings of the first kind, the oblique tilings, are periodic in the sense that they are invariant under the action of the discrete subgroup $\mathbf{Z}^{n}$ of $\mathbf{R}^{n}$. The oblique tiles depend on two orthogonal complementary subspaces $E$, of dimension $d$, and $E^{\prime}$ of $\mathbf{R}^{n}$ which are given a priori with an arbitrary, albeit fixed, orientation with respect to the lattice. Every oblique tile a "rectangle," that is the direct sum of a polyhedron in $E$ and a polyhedron in $E^{\prime}$; the polyhedron in $E$ is the projection (onto $E$ ) of a $d$-dimensional facet (a $d$-facet, for short) of the $n$-dimensional unit cube; the polyhedron in $E^{\prime}$ is, similarly but up to a sign, the projection (onto $E^{\prime}$ ) of the $(n-d)$ facet which is complementary to the previous one. If one proceeds in this way for all the $\left(\begin{array}{l}n \\ d\end{array}\right)$ differently oriented facets having the same dimension as $E$, one builds a set of $\left(\begin{array}{l}n \\ d\end{array}\right)$ oblique polytopes in $\mathbf{R}^{n}$ which form a partition of a fundamental cell for $\mathbf{Z}^{n}$ and thereby provide a set of so-called "prototiles" for the tiling. This is stated and proved in our main theorem in Sect. III. 
Preliminary, contingent, versions of our "oblique" tiling appeared in the literature dealing with the theory of Anosov diffeomorphisms. Being periodic, the oblique tiling yields, by projection onto the quotient, a finite partition of the $n$-torus $\mathbf{T}^{n}=\mathbf{R}^{n} / \mathbf{Z}^{n}$. Now, starting from a linear hyperbolic operator $h$ on $R^{n}$ satisfying $h \mathbf{Z}^{n}=\mathbf{Z}^{n}$, one obtains a diffeomorphism $f$ of the torus by projecting $h$ onto $\mathbf{T}^{n}$ : this is a standard example of diffeomorphisms of Anosov's type. For such diffeomorphisms in dimension $n=2$, Adler and Weiss [1] constructed Markov partitions (see for ex. [2]) and their result, when lifted back into $\mathbf{R}^{2}$, is an oblique tiling with $E$ and $E^{\prime}$ identified to, respectively, the stable and unstable eigenspaces of $h$. Thus, when this identification is done, the oblique tiling projects precisely onto a Markov partition for the induced $f$.

In $n=3$ dimensions, the Markov partitions are not smooth [3]. Nevertheless, explicit Markov partitions are available for such diffeomorphisms on $\mathbf{T}^{3}$ as provided by Bedford's work using fractals [4]. In his procedure generating fractal Markov partitions, the starting point is a 3-dimensional tiling with three prototiles which turns out to be a particular realization of our oblique tiling ${ }^{1}$.

Those two examples lead us to address the question of whether the object common to the $n=2$ and $n=3$ cases, namely the oblique tiling, exists in arbitrary dimension and whether its fundamental property - to tile $\mathbf{R}^{n}$ - can be given direct statement and proof in affine geometry. A positive answer to both questions is given in the course of this paper (Sect. III). Moreover, whereas the quoted Markov partitions involve only a particular set of subspaces - those which are eigenspaces of unimodular hyperbolic matrices with integer coefficients - the geometrical construction of the oblique tiling remains valid for arbitrary orientations of the pair $E, E^{\prime}$.

Another application, to which the present paper aims to contribute more extensively, deals with the crystallography of quasicrystals. Affine cuts of the oblique tiling along parallel translates of $E$ yield regular tilings of $E$; moreover those tilings are quasiperiodic in generic situations ${ }^{2}$. Quasiperiodic tilings provide tentative templates to describe the order of the atomic constituent in the quasicrystalline phases of metallic compounds such as AlMn, AlMnSi, AlLiCu, and others $[5,6]$. Because of discreteness - a tiling is characterized by a finite number of prototiles; here, all the prototiles shall be polyhedra -- such templates take into account the finite size of the inter-atomic distances. Moreover, as shown in a number of models, quasiperiodic ordering satisfactorily fits to the long range order of such phases. This is established on the basis that the Fourier transform of the tilings agrees with the diffraction spectra (neglecting effects such as the enlargement of the spots due to disorder, structural defects, etc.). The diffraction

\footnotetext{
${ }^{1}$ Bedford's proof that he obtains a true tiling seems to rely upon the one-dimensionality of the contracting subspace (say $E^{\prime}$ ) meaning that the complementary $E$ is a splitting (hyper-)plane, a property which does not hold in general

${ }^{2}$ The quasiperiodic tilings constructed in this way are denominated canonical - refering to the standard unit cube at the heart of the construction - to keep clear that there are many other quasiperiodic tilings which are neither canonical nor obtained from canonical ones by local decorations or transformations. In this paper, since we are not concerned with any of these other t1lings, quasiperiodic tilings always means canonical, i.e. cut of an oblique one, unless otherwise stated (see Sect. VII)
} 
peaks are contained in a finitely generated module over the integers $\mathbf{Z}$; in cases involving irrational commensurations, such a spectrum would be a dense subset of $E$ if there was no sensibility edge in the detector.

Usually, underlying $\mathbf{Z}$-modules in the diffraction as well as Fourier spectra are considered as characteristic of quasiperiodic ordering. Several algorithms have been proposed to build quasiperiodic models: the dual grid method [7, 8], Janner and Jansen's section method [9,10], and the strip or projection method [11]; in a number of cases including the canonical quasiperiodic tilings, these methods are equivalent (see Sects. IV, V and [12]). The last two methods generate discrete sets of sites (in crystallographic models, these sites may be atomic sites or the sites of structural units composed of several atoms). However, questions about the local order or the inference of the global from the local order often arise in ill-stated manners due to a lack of direct proofs that the above quoted methods actually generate true tilings. In the present paper, this problem is solved, we prove that the strip algorithm leads to an authentic tiling. To do so, in Sect. VI, we show that a fundamental conjecture underlying the strip algorithm (see e.g. [13] p. 192) holds true, namely that the strip selects a unique simply connected faceted manifold which projects onto the tiling. On the other hand, Janner and Jansen's technique provides the set of the vertices of the tiling in question (Sect. V) because the periodic set of "vertical" surfaces (or atomic surfaces) entering this algorithm proves out to be a part of the $(n-d)$-dimensional boundary of the oblique tiling.

As to the dual grid method, a proof that it yields a tiling was given by de Bruijn in [14] where he constructs a continuous differentiable function approximating the tiling and he applies Hadamard's theorem about differentiable maps with nonvanishing Jacobian ${ }^{3}$. Our proof, on its side, is immediate and uses only simple (n-dimensional) geometry and linear algebra. A crucial step consists in building the $n$-dimensional oblique tiling. Then, the quasiperiodic tilings are obtained as plain affine sections of the oblique one.

This method sheds light on the quasiperiodic ordering of such tilings. A function is quasiperiodic if it is the affine section (meant here as the restriction to an affine subspace) of a continuous periodic function defined in higher dimensions. This property goes back to the pioneering works on quasiperiodicity by Bohr [15] and Besicovitch [16]. With some care, such a characterization of quasiperiodicity extends to discrete subsets of $E$. For example, consider the $\mathbf{Z}^{n}$ orbit of a bounded regular domain $K$ of $E^{\perp}$ in $\mathbf{R}^{n}$. Then a cut, by $E$, through this periodic set generates a discrete set which, by construction, may be thought of as quasiperiodic. This is Janner and Jansen's description of modulated crystals [17] and Penrose tilings [9]. The further extension needed to get a whole tiling rather than only a discrete set - such as the vertices - as a cut through a periodic structure is developed in Sects. III and IV below. Concerning quasicrystals, similar ideas have recently been presented by P. Kramer in some particular cases [18].

In applications to crystallography, the choice of the embedding dimension $n$ and of the orientation of $E$ are prescribed by symmetry $[11,13]$. The resulting

\footnotetext{
${ }^{3}$ According to the equivalence there is between the various techniques [7], one could, in principle, refer to de Bruijn's proof to set down that the strip method yields a tiling. This way is, however, a fairly indirect detour
} 
quasiperiodic tiling will be periodic in the directions spanned by the intersection $E \cap \mathbf{Z}^{n}$ and aperiodic in the complementary ones (in $E$ ). The famous Penrose tilings [19], for example, involve 5-fold rotations, whereas 3-dimensional quasicrystals involve point symmetries like the icosahedron group, 8 or 12 -fold rotations. For further references on point symmetries in quasicrystals, see Rokhsar et al. [20] and Cartier [21]. The argument we present, however, does not depend on any particular point symmetry: only the translations are involved in analysing the (quasi-)periodic order.

In summary, the basic definitions and notations are collected in Sect. II. The oblique tiling is constructed in Sect. III, devoted to demonstrate that this tiling tiles $\mathbf{R}^{n}$. That generic affine sections of the oblique tiling provide quasiperiodic tilings of the subspaces $E$ is stated in Sect. IV. What happens for non-generic sections (such cases are also called singular) is then discussed. In Sect. V is shown that the $J-J$ section procedure provides the vertices of the quasiperiodic tiling. The projection method is revisited in Sect. VI: we prove that the strip contains a unique simply connected faceted surface which projects bijectively onto the tiling. The last section, Sect. VII, is devoted to some prospective and conclusions.

The Sects. I-IV are of general interest in the theory of tilings, providing the settings and verifying the basic properties. On the other hand, the Sects. V and VI are more technical, relating our section algorithm to previously known methods: we aim to provide a synthetic picture and (new) proofs to some fundamental conjectures.

\section{Definitions and Settings}

1. A subset $A$ of $\mathbf{R}^{n}$ is regular if it is bounded with non-empty interior $\operatorname{Int}(A)$ and such that its closure $\operatorname{Clos}(A)$ coincides with $\operatorname{Clos}(\operatorname{Int}(A))$ and its interior $\operatorname{Int}(A)$ equals $\operatorname{Int}(\operatorname{Clos}(A))$. The topology is provided by the euclidean norm $\|x\|=\left(\sum x_{i}^{2}\right)^{1 / 2}$ for $x=\left(x_{1}, \ldots, x_{n}\right) \in \mathbf{R}^{n}$.

In this work, the regular sets will be polygons in $d=2$, polyhedra in $d=3$ dimensions, or polytopes in higher dimensions. The theory is presented in terms of polytopes to avoid unnecessarily complicated writings but, even if we don't mention it systematically, many of our assertions remain true for regular sets which are not polytopes.

2. A partition of a topological space $E$ is a covering of $E$ by disjoint sets. If the sets are regular, we say that the partition is regular. In other words, a regular partition is a set $P$ of regular subsets of $E$ satisfying:

a)

$$
\operatorname{Clos}\left(\bigcup_{A \in P} A\right)=E
$$

b)

$$
\operatorname{Int}(A) \cap \operatorname{Int}(B)=\emptyset, \quad \forall A, B \in P, A \neq B .
$$

The elements of $P$ are called domains.

3. Let $E$ be a topological vector space. Two subsets $A$ and $B$ of $E$ are congruent (through the translations) if there is a translation $t$ such that $A+t=B$. Congruence defines an equivalence relation among the domains of any partition $P$. 
When the number of equivalence classes - the congruence classes modulo translations - is finite, the partition is a tiling. The corresponding domains are the tiles and we call the congruence classes prototiles. (As usual, we often use the same word - prototile - for the congruence class and for any prototype representing this class.) Thus, if $T$ denotes the translation group, a partition $P$ is a tiling if $P / T$ is finite.

4. Throughout the text, $E, E^{\prime}$ will be a pair of orthogonal complementary subspaces of $\mathbf{R}^{n}$. To the decomposition $\mathbf{R}^{n}=E \oplus E^{\prime}$ corresponds the pair of orthogonal projectors $\pi$, with range $E$, and $\pi^{\prime}=1-\pi$, with range $E^{\prime}$; the dimensions are $\operatorname{dim}(E)=d$ and $\operatorname{dim}\left(E^{\prime}\right)=d^{\prime}=n-d$.

5. $\left\{\varepsilon_{1}, \ldots, \varepsilon_{n}\right\}$ denotes the standard orthonormal basis of the $n$-dimensional Euclidean space $\mathbf{R}^{n} . \Lambda=\mathbf{Z}^{n}$ is the simple hypercubic lattice of $\mathbf{R}^{n}$ generated by $\left\{\varepsilon_{1}, \ldots, \varepsilon_{n}\right\}$. The canonical fundamental domain for $\mathbf{Z}^{n}$ in $\mathbf{R}^{n}$ is the unit hypercube $\gamma_{n}$ in $n$ dimensions:

$$
\gamma_{n}=\left\{\sum_{i=1}^{n} \lambda_{i} \varepsilon_{i} \mid \lambda_{i} \in[0,1] \text { for } i=1, \ldots, n\right\} .
$$

6. Let $p$ be an integer $0 \leqq p \leqq n$ and $M_{p}=\left\{I=\left\{i_{1}, \ldots, i_{p}\right\} \subset\{1, \ldots, n\}\right\}$ be the set of subsets of $\{1, \ldots, n\}$ with $p$ elements. The $p$-facets of the unit cube are indexed by $M_{p}$ as follows:

$$
\begin{aligned}
& \gamma_{I}=\left\{\sum_{i \in I} \lambda_{i} \varepsilon_{i} \mid \lambda_{i} \in[0,1]\right\} \text { for all } I \in M_{p} \text { and } p>0, \\
& \gamma_{\emptyset}=\{0\} .
\end{aligned}
$$

If $I^{c}=\{1, \ldots, n\} \backslash I \in M_{n-p}$ is the complement of $I \in M_{p}$, the unit cube admits the decomposition ${ }^{4}$

$$
\gamma_{n}=\gamma_{I}+\gamma_{I^{c}} .
$$

Moreover, $\pi_{I}$ is the projector onto the coordinate subspace spanned by $\{\varepsilon\}_{I}=\left\{\varepsilon_{i} \mid i \in I\right\}$.

7. The projections $L=\pi(\Lambda)$ and $L^{\prime}=\pi^{\prime}(\Lambda)$ of $\Lambda$ in $E$ et $E^{\prime}$ are $\mathbf{Z}$-modules generated respectively by $\left\{e_{1}, \ldots, e_{n}\right\}$ and $\left\{e_{1}^{\prime}, \ldots, e_{n}^{\prime}\right\}$, where $e_{i}=\pi\left(\varepsilon_{i}\right)$ and $e_{i}^{\prime}=\pi^{\prime}\left(\varepsilon_{i}\right)$. If we set $\Lambda_{E}=\Lambda \cap E$ and $\Lambda_{E^{\prime}}=\Lambda \cap E^{\prime}, L$ and $L^{\prime}$ are isomorphic to $\Lambda / \Lambda_{E^{\prime}}$ and $\Lambda / \Lambda_{E}$ respectively. When $\Lambda_{E}$ reduces to $\{0\}$, the orientation of $E$ with respect to $A$ is said to be totally irrational or incommensurate. Otherwise, $\Lambda_{E}$ and $\Lambda_{E^{\prime}}$ are non-trivial sublattices of $\Lambda$.

Generically, the orientation is irrational. In this case, the systems $\left\{e_{1}, \ldots, e_{n}\right\}$ and $\left\{e_{1}^{\prime}, \ldots, e_{n}^{\prime}\right\}$ are independent on $\mathbf{Z}$ (and thus on $\mathbf{Q}$ ) and $\Lambda$ is isomorphic to each of its projections $L$ and $L^{\prime}$. Conversely, any non-trivial relation $\sum n_{i} e_{i}=0$ (respectively $\sum n_{i} e_{i}^{\prime}=0$ ), with $n_{i} \in \mathbf{Z}$, implies $\sum n_{i} \varepsilon_{i} \in \Lambda_{E^{\prime}}$ (respectively $\Lambda_{E}$ ) and corresponds to an algebraic equation satisfied by $\pi$.

${ }^{4}$ For $A$ and $B C \mathbf{R}^{n}$, we write $A+B$ for $\{a+b \mid a \in A, b \in B\}$ 
8. We shall assume the following property (which is also generic):

Non-degeneracy hypothesis: For any $I=\left\{i_{1}, \ldots, i_{d}\right\} \in M_{d}$, the system $\{e\}_{I}=\left\{e_{i_{1}}, \ldots, e_{i_{d}}\right\}$ is of rank $d$ in $E$ and the system $\left\{e^{\prime}\right\}_{I^{c}}=\left\{e_{i}^{\prime} \mid i \in I^{c}\right\}$ is of rank $n-d$ in $E^{\prime}$.

Actually, in our setting where $\pi$ is orthogonal, the two statements mentioned in the hypothesis are equivalent; the ranks of $\{e\}_{I}$ and $\left\{e^{\prime}\right\}_{I^{c}}$ are simultaneously maximal or not maximal as shown in

Lemma II.1. Let $p$ be an integer $0 \leqq p \leqq n$ and let $I \in M_{p}$. The ranks $r$ of $\{e\}_{I}$ and $r^{\prime}$ of $\left\{e^{\prime}\right\}_{I^{c}}$ satisfy $r^{\prime}=n-d-p+r$.

Proof. If $r \leqq p$ is the rank of $\{e\}_{I}$ in $E$, there exist $p-r$ independent linear relations $\sum_{i \in I} \lambda_{j, i} e_{i}=0,(j=1, \ldots, p-r)$. Thus the $p-r$ vectors $\sum_{i \in I} \lambda_{j, i} \varepsilon_{i}(j=1, \ldots, p-r)$ are independent in $\mathbf{R}^{n}$ and coincide with their projection on $E^{\prime}$. Now, for any $k \in I^{c}$ and any $j=1, \ldots, p-r$, we have (using the orthogonality of $\pi$ ):

$$
0=\sum_{i \in I} \lambda_{j, i}\left(\varepsilon_{i}, \varepsilon_{k}\right)=\sum_{i \in I} \lambda_{j, i}\left(e_{i}^{\prime}, e_{k}^{\prime}\right) .
$$

Thus the system $\left\{e_{k}^{\prime} \mid k \in I^{c}\right\}$ is orthogonal to $E$ and to $p-r$ independent vectors of $E^{\prime}$; therefore its rank satisfies $r^{\prime} \leqq n-d-p+r$. A symmetric argument implies $r \leqq d-(n-p)+r^{\prime} . \quad$ QED

The 3-dimensional rombohedral tilings involve a construction in $\mathbf{R}^{6}$ where the orientation of $E$ (and $E^{\prime}$ ) is completely specified by the icosahedral symmetry. The above condition is satisfied since the projections of the 3 -facets of $\mathbf{Z}^{6}$ are rhombohedra [11].

\section{Oblique Periodic Tilings of $\mathbf{R}^{\boldsymbol{n}}$}

In this section we present the construction of the "oblique" tilings of $\mathbf{R}^{n}$ associated to a pair $E, E^{\prime}$ of orthogonal complementary subspaces.

When the non-degeneracy hypothesis II.8 is satisfied, the $d$-facets of $A$ are isomorphic to their projections on $E$ by $\pi$; similarly the $d^{\prime}$-facets of $\Lambda$ are isomorphic to their projections on $E^{\prime}$.

Definitions III.1. For any subset $I \subset\{1, \ldots, n\}$, let

$$
D_{I}=\pi\left(\gamma_{I}\right)=\left\{\sum_{i \in I} \lambda_{i} e_{i} \mid \lambda_{i} \in[0,1]\right\} \quad \text { and } \quad \mathrm{D}_{I}^{\prime}=\pi^{\prime}\left(\gamma_{I}\right)=\left\{\sum_{i \in I} \lambda_{i} e_{i}^{\prime} \mid \lambda_{i} \in[0,1]\right\}
$$

be the projections of the facet $\gamma_{I}$ on $E$ and $E^{\prime}$.

Define the rectangular polytope $\Delta_{I}$ as

$$
\Delta_{I}=D_{I}-D_{I^{c}}^{\prime}=\left\{x-x^{\prime} \mid x \in D_{I}, x^{\prime} \in D_{I^{c}}^{\prime}\right\},
$$

and set $\Delta_{I, \xi}=\Delta_{I}+\xi$, where $\xi \in \mathbf{Z}^{n}$.

We assert:

Theorem III.1. The set $\mathbb{D}=\left\{\Delta_{I, \xi} \mid I \in M_{d}\right.$ and $\left.\xi \in \mathbf{Z}^{n}\right\}$ is a periodic tiling of $\mathbf{R}^{n}$, the unit cell of which is the disjoint union of the $\left(\begin{array}{l}d \\ n\end{array}\right)$ subcells $\Delta_{I}$. 
The proof is divided in two propositions: first we prove, by standard multilinear algebra, that these subcells have a total volume equal to $1\left(=\operatorname{vol}\left(\gamma_{n}\right)\right)$, and we shall prove, next, that different subcells do not overlap.

Proposition III.2. For any $I=\left\{i_{1}, \ldots, i_{d}\right\} \subset\{1, \ldots, n\}$, let $V_{I}$ and $V_{I^{c}}^{\prime}$ denote the volumes of $D_{I}$ and $D_{I^{c}}^{\prime}$ (so that the volume of $\Delta_{I}$ is $V_{I} \cdot V_{I^{c}}^{\prime}$ ). Then

$$
\sum_{I \in M_{d}} V_{I} \cdot V_{I^{c}}^{\prime}=1
$$

Proof. Let $\varepsilon_{1} \wedge \ldots \wedge \varepsilon_{n}$ denote the wedge product of the basis vectors of $\mathbf{R}^{n}$. The decomposition $\varepsilon_{i}=e_{i}+e_{i}^{\prime}$ yields by multilinearity

$$
\varepsilon_{1} \wedge \ldots \wedge \varepsilon_{n}=\sum s(I) e_{I} \wedge e_{I^{c}}^{\prime},
$$

where $s(I)$ is the signature of the permutation

$$
(1, \ldots, n) \rightarrow\left(I, I^{c}\right)=\left(i_{1}, \ldots, i_{d}, i_{d+1}, \ldots, i_{n}\right), \quad e_{I}=e_{i_{1}} \wedge \ldots \wedge e_{i_{d}}
$$

and

$$
e_{I^{c}}^{\prime}=e_{i_{d+1}}^{\prime} \wedge \ldots \wedge e_{i_{n}}^{\prime} .
$$

In (3.2), use is made of the fact that the wedge product of $k$ vectors vanishes when $k$ exceeds their rank.

By definition of the volumes,

$$
e_{I} \wedge e_{I^{c}}^{\prime}=\sigma_{I} \cdot V_{I} \cdot V_{I^{c}}^{\prime} \cdot \varepsilon_{I} \wedge \varepsilon_{I^{c}}
$$

with $\sigma_{I}= \pm 1$. Using again $\varepsilon_{i}=e_{i}+e_{i}^{\prime}$ for $i \in I^{c}$, we obtain $e_{I} \wedge e_{I^{c}}^{\prime}=e_{I} \wedge \varepsilon_{I^{c}}$. By $e_{1}=\pi_{I}\left(e_{i}\right)$ for $i \in I$ (in the writings II.6), we obtain

$$
e_{I} \wedge \varepsilon_{I^{\mathrm{c}}}=\operatorname{det}(\pi[I]) \cdot \varepsilon_{I} \wedge \varepsilon_{I^{\mathrm{c}}},
$$

$\pi[I]$ being the minor of $\pi$ with indices in $I \times I$. Now, since $\pi$ is a positive symmetric operator, the partial determinant is non-negative, proving that $\sigma_{I}=+1$. The proof is achieved by introducing (3.3) into (3.2). QED

Remark. It follows from Lemma II.1 that, for any $I=\left\{i_{1}, \ldots, i_{d}\right\}$, either $V_{I} \cdot V_{I^{c}}^{\prime} \neq 0$ or $V_{I}=V_{I^{c}}=0$.

After the former proposition, showing that the set $\mathbb{O}=\left\{\Delta_{I, \xi} \mid I \in M_{d}\right.$ and $\left.\xi \in \mathbf{Z}^{n}\right\}$ is a tiling of $\mathbf{R}^{n}$ amounts to check the non-overlapping property (2.2).

Proposition III.3. Let $\Delta_{I . \xi}$ be as in Definition III.1. Then, for any $I, J \in M_{d}$ and any $\xi \in \mathbf{Z}^{n}$, either the interiors of $\Delta_{I}$ and of $\Delta_{J}+\xi$ are disjoint, or $I=J$ and $\xi=0$.

Proof. Let $I=\left\{i_{1}, \ldots, i_{d}\right\}, J=\left\{j_{1}, \ldots, j_{d}\right\}$. Suppose that there is a point $a$ in $\Delta_{I} \cap\left(\Delta_{J}+\xi\right)$. This means that there exist $\lambda_{1}, \ldots, \lambda_{n}$ and $\mu_{1}, \ldots, \mu_{n}$, all in $[0,1]$, such that:

$$
a=\sum_{i \in I} \lambda_{i} \cdot e_{i}-\sum_{i \in I^{c}} \lambda_{i} \cdot e_{i}^{\prime}=\sum_{j \in J} \mu_{j} \cdot e_{j}-\sum_{j \in J c} \mu_{j} \cdot e_{j}^{\prime}+\xi .
$$

Projecting on $E$ and $E^{\prime}$ yields:

$$
\sum_{i \in I} \lambda_{i} \cdot e_{i}-\sum_{j \in J} \mu_{j} \cdot e_{j}-x=0,
$$


where $x=\pi(\xi)$,

$$
-\sum_{i \in I^{c}} \lambda_{i} \cdot e_{i}^{\prime}+\sum_{j \in J^{c}} \mu_{j} \cdot e_{j}^{\prime}-x^{\prime}=0
$$

and $x^{\prime}=\pi(\xi)$. Define

$$
u=\sum_{i \in I} \lambda_{i} \cdot \varepsilon_{i}-\sum_{j \in J} \mu_{j} \cdot \varepsilon_{j}-\xi
$$

and

$$
v=-\sum_{i \in I^{c}} \lambda_{i} \cdot \varepsilon_{i}+\sum_{j \in J^{c}} \mu_{j} \cdot \varepsilon_{j}-\xi .
$$

Since $\pi(u)=0$ and $\pi^{\prime}(v)=0$, we have $u \in E^{\prime}$ and $v \in E$; in particular, the scalar product $(u, v)$ vanishes.

If $\xi=\left(\xi_{1}, \ldots, \xi_{n}\right)$, we get:

$$
\begin{aligned}
0= & (u, v)=\sum_{k \in I \backslash J}\left[\lambda_{k} \mu_{k}-\xi_{k}\left(\lambda_{k}+\mu_{k}\right)+\xi_{k}^{2}\right]+\sum_{k \in J \backslash I}\left[\lambda_{k} \mu_{k}+\xi_{k}\left(\lambda_{k}+\mu_{k}\right)+\xi_{k}^{2}\right] \\
& +\sum_{k \in I \cap J}\left[\xi_{k}\left(-\lambda_{k}+\mu_{k}\right)+\xi_{k}^{2}\right]+\sum_{k \in I^{c} \cap J^{c}}\left[\xi_{k}\left(\lambda_{k}-\mu_{k}\right)+\xi_{k}^{2}\right], \\
0= & \sum_{k \in I \backslash J}\left(\xi_{k}-\lambda_{k}\right)\left(\xi_{k}-\mu_{k}\right)+\sum_{k \in J \backslash I}\left(\xi_{k}+\lambda_{k}\right)\left(\xi_{k}+\mu_{k}\right) \\
& +\sum_{k \in I \cap J} \xi_{k}\left(\xi_{k}-\lambda_{k}+\mu_{k}\right)+\sum_{k \in I^{c} \cap J^{c}} \xi_{k}\left(\xi_{k}+\lambda_{k}-\mu_{k}\right) .
\end{aligned}
$$

One easily checks that the conditions $0 \leqq \lambda_{k}, \mu_{k} \leqq 1$ imply that each term, considered as a second degree polynomial in $\xi_{k}$, is larger than or equal to 0 as soon as $\xi_{k}$ is an integer. Thus $(u, v)=0$ implies the vanishing of each term of (3.4); now,

$$
\begin{gathered}
\left(\xi_{k}-\lambda_{k}\right)\left(\xi_{k}-\mu_{k}\right)=0 \text { implies } \xi_{k}=\lambda_{k} \text { or } \xi_{k}=\mu_{k}, \\
\left(\xi_{k}+\lambda_{k}\right)\left(\xi_{k}+\mu_{k}\right)=0 \text { implies } \xi_{k}=-\lambda_{k} \text { or } \xi_{k}=-\mu_{k}, \\
\xi_{k}\left(\xi_{k}-\lambda_{k}+\mu_{k}\right)=0 \text { implies } \xi_{k}=0 \text { or } \xi_{k}=\lambda_{k}-\mu_{k}=-1,0 \text { or } 1, \\
\xi_{k}\left(\xi_{k}+\lambda_{k}-\mu_{k}\right)=0 \text { implies } \xi_{k}=0 \text { or } \xi_{k}=-\lambda_{k}+\mu_{k}=-1,0 \text { or } 1 .
\end{gathered}
$$

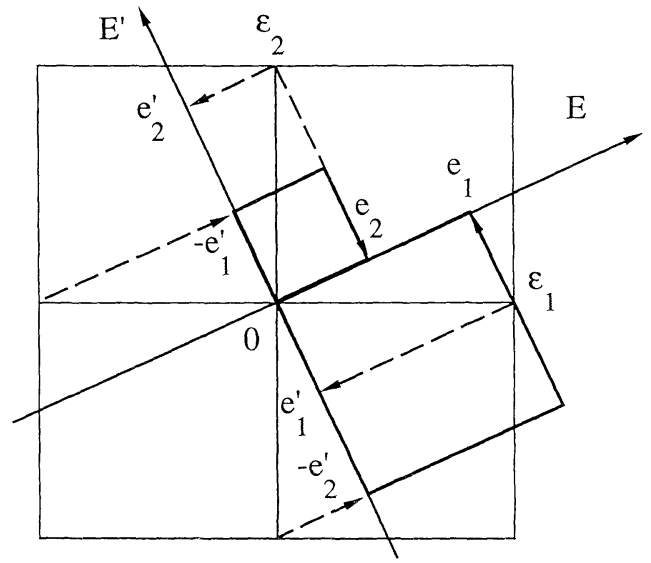

Fig. 1. Construction of the unit cell of the oblique tiling in the $n=2$-dimensional case 
An equation of the type $\xi_{k}=\lambda_{k}$ means that both $\xi_{k}$ and $\lambda_{k}$ are 0 or 1 ; a similar analysis applied to the four equations covers all the possible cases. Therefore, in any case, we conclude that, when it exists, an intersection point a necessarily belongs to the boundary of one of the two subcells $\Delta_{I}$ or $\Delta_{J}+\xi$, unless $\xi=0$ and $I=J$. QED

Remark. It follows from (3.4-5) that intersection points $a \in \Delta_{I} \cap\left(\Delta_{J}+\xi\right)$, which are now contact points, exist only when $\left|\xi_{k}\right| \leqq 1$ for all $k=1, \ldots, n$.

\section{Quasiperiodic Tilings}

An immediate property of the oblique tiling is that any section of it along a subspace parallel to $E$ actually defines a tiling of the subspace. As we argue in this section this is true for almost all parallel cuts, but a tighter analysis shows that, in a non-generic set of such cases, the section tiling is only defined with some ambiguities. Singular sections are briefly studied.

Let $E+t$ be an affine subspace of $\mathbf{R}^{n}$; the translation $t$ belongs to $\mathbf{R}^{n} / E$ which is identified to $E^{\prime}$. Suppose $\mathbf{R}^{n}$ is provided with a tiling $\mathbb{T}$; we call an $E$-section of $\mathbb{T}$ the partition which is induced, on $E$, by the intersection of $E+t$ with the $n$-dimensional tiles:

$$
((E+t) \cap \mathbb{T})-t=E \cap(\mathbb{T}-t) .
$$

The singular situations occur when the cutting subspace $E+t$ intersects the boundary of a cell of $\mathbb{T}$ over a domain with non-empty interior (in $E+t$ ). From the definition of the oblique tiling in Sect. III, the boundary, defined by $\partial \Phi=\left\{\partial \Delta_{I, \xi}\right\}$, may be split in two parts:

$$
\partial \boldsymbol{D}=\partial^{\left(E^{\prime}\right)} \boldsymbol{D} \cup \partial^{(E)} \boldsymbol{D},
$$

with

$$
\begin{aligned}
& \partial^{(E)} \mathbb{D}=\left\{D_{I}-\partial D_{I^{c}}^{\prime}+\xi \mid(I, \xi) \in M_{d} \times \mathbf{Z}^{n}\right\}, \\
& \partial^{\left(E^{\prime}\right)} \mathbb{D}=\left\{\partial D_{I}-D_{I^{c}}^{\prime}+\xi \mid(I, \xi) \in M_{d} \times \mathbf{Z}^{n}\right\} .
\end{aligned}
$$

Since $\partial^{\left(E^{\prime}\right)} \Phi$ is transverse to $E$, the singular situations correspond to

$$
t \in T_{0}=\pi^{\prime}\left(\partial^{(E)}(\mathbf{D}) .\right.
$$

By the fact that $D_{I} \subset \operatorname{ker} \pi^{\prime}$, the definition of $L^{\prime}$ in II.7, and a change of index in the union, this singular set may be written as

$$
T_{0}=L^{\prime}-\bigcup_{I \in M_{d}} \partial D_{I}^{\prime} .
$$

Being a countable union of bounded affine manifolds of codimension 1 , the singular set (4.4) has Lebesgue measure zero in $E^{\prime}$.

In regular situations, $t \notin \pi^{\prime}\left(\partial^{(E)} \mathbb{D}\right)$, the intersection $(E+t) \cap \Delta_{I, \xi}$ is either empty or equal to $D_{I}+\pi(\xi)+t$. This, together with the fact that a regular $E$-section of a tiling partitions $E$, leads to the following statement: 
Proposition IV.1. Any regular E-section of the oblique tiling is a tiling 5 :

$$
\mathbb{P}_{t}=E \cap(\mathbb{Q}-t) \text {. }
$$

The set of prototiles of the section is the set of projected $d$-facets $D_{I}=\pi\left(\gamma_{I}\right)$ for $I \in M_{d}$.

The number of prototiles is bounded by $\left(\begin{array}{l}d \\ n\end{array}\right)$. In some cases, this number may be reduced by additional symmetry. If the congruence relation among tiles is considered not only with respect to pure translations, but also with respect to the full euclidean group acting on $E$, including rotations, then the rotational symmetry may enlarge the congruence classes, and therefore reduce the number of prototiles. For example, the $d=2$ dimensional generalized Penrose tilings are $E$-sections of a 5-dimensional oblique tiling where the subspace $E$ is spanned by the two following vectors, written in the canonical basis $\left(\varepsilon_{1}, \ldots, \varepsilon_{5}\right)$ :

$$
\begin{aligned}
& v_{1}=(1, \cos 2 \pi / 5,-\cos \pi / 5,-\cos \pi / 5, \cos 2 \pi / 5), \\
& v_{2}=(0, \sin 2 \pi / 5, \sin \pi / 5,-\sin \pi / 5,-\sin 2 \pi / 5) .
\end{aligned}
$$

In this case, the prototiles are the two Penrose rhombi.

Similarly, the icosahedral tilings in $d=3$ dimensions with two prototiles result as $E$-sections of the 6-dimensional oblique tiling, under the condition that $E$ is the range of the projector given, for ex., on p. 184 of [13].

The identity between these two examples and their respective sections of oblique tilings will become clear in the next sections where we relate our section procedure to the previously known methods generating Penrose or icosahedral tilings.

Comment IV.1. In general, sections of periodic tilings are partitions with an infinite number of non-congruent domains. For example, irrational cuts of the simple hypercubic tiling generated by the $\mathbf{Z}^{n}$ orbit of the unit cube $\gamma_{n}$ partition the section in domains which are all different ${ }^{6}$. If one wishes to obtain a true tiling in the cut (with a finite number of prototiles), one may be proceed in two different ways:

i) By letting $E$ be a lattice subspace; then the $E$-section of any periodic tiling is a periodic partition of $E$, therefore a tiling.

ii) If $E$ is not a lattice subspace, what happens in the generic cases and in the examples involving 5-fold symmetries, we may choose, among all the possible fundamental cells for the lattice, a special one inducing a tiling in the section.

Alternative b) prevails in the present work. The oblique tiling, in Sect. III, has been constructed in this perspective. The following picture illustrates the situation: Singular Sections. For singular positions of $E+t$, say $t=t_{0} \in T_{0}$, the section is not properly defined. By hypothesis, the region

$$
W=E \cap\left(\partial^{(E)} \mathbb{D}-t_{0}\right)
$$

has a non-empty interior in $E$ but the section fails to prescribe, in an unambiguous way, how this region ought to be covered. To see this, let us consider a bounded

\footnotetext{
${ }^{5}$ The tiling $\mathbb{P}$ is referred to as the quasiperiodic tiling (see note 2). Of course, quasiperiodicity doesn't forbid periodicity, a priori

${ }^{6}$ The boundaries of this partition constitute a so-called multigrid
} 

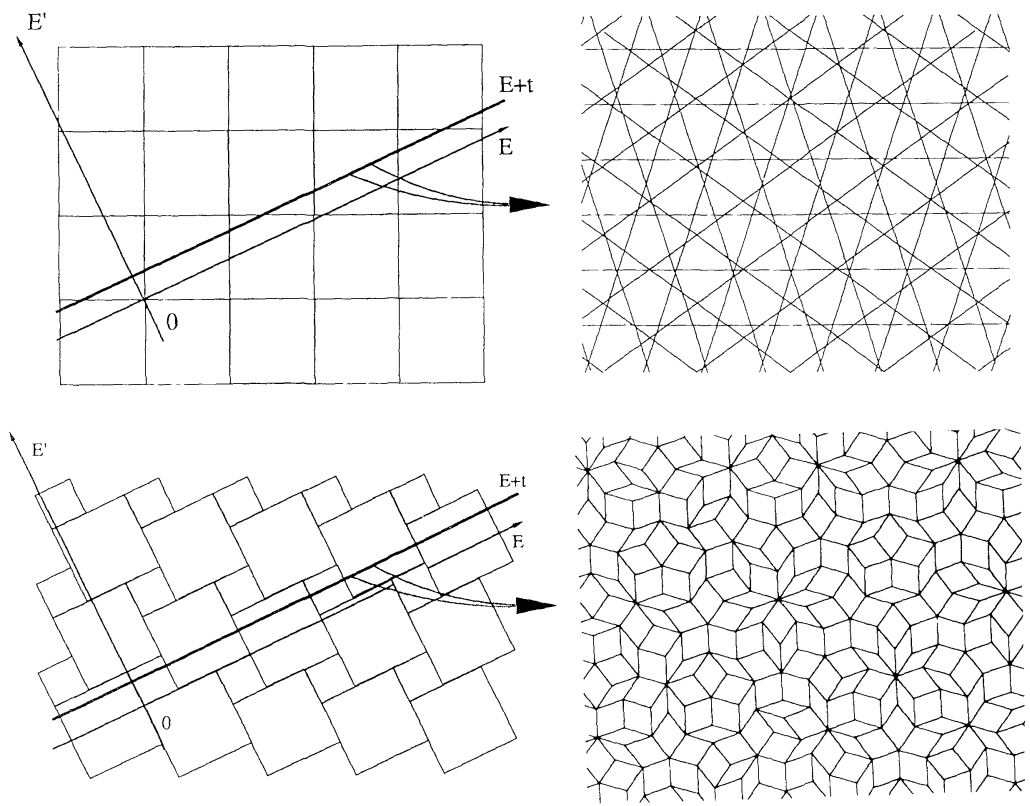

Fig. 2. E-sections of the standard cubic tiling (top) and of the oblique tiling (bottom)

region $B C E$ which is large enough to cover a significant part of $W$ (in general, $W$ is a discrete union of bounded connected components: suppose that $B$ contains at least one of these components). Consider, also, the mapping

$$
t \rightarrow B \cap(\mathbb{D}-t),
$$

defined in a neighborhood $N\left(t_{0}\right)$ of $t_{0}$ in $E^{\prime}$. This application maps points around $t_{0}$ into tilings of $B$ in a piecewise constant way. Now, since $W \cap B$ spreads over facets lying in the interface of two or more cells $\Delta_{I, \xi}, t_{0}$ is a discontinuity point of this mapping.

Despite its non-genericity, the singular set may be a large subset of $\mathbf{R}^{n}$. Indeed, for an irrationally oriented $E$, this set is a dense stacking of planes of codimension 1 in $E^{\prime}$ :

Lemma IV.2. Let $\mathbb{D}$ be the oblique tiling of Sect. III. For $J \subset\{1, \ldots, n\}$ denote by $E_{J}^{\prime}$ the plane spanned by $\left\{e^{\prime}\right\}_{J}=\left\{e_{i}^{\prime} \mid i \in J\right\}$. Then

$$
T_{0}=L^{\prime}+\bigcup_{|J|=d^{\prime}-1} E_{J^{\prime}}
$$

Proof. Equation (4.4) states that $T_{0}$ is contained in the right-hand side of (4.8). To prove the inclusion in the other direction, note that $\partial D_{I}^{\prime}$ is the projection of the boundary of the $d^{\prime}$-facet $\gamma_{I}$, which itself is a union of (translated) facets $\gamma_{J}$, with $|J|=d^{\prime}-1=|I|-1$. Now $\bigcup_{I}\left(L^{\prime}+\partial D_{I}^{\prime}\right)$ contains $L_{J}^{\prime}+\pi^{\prime}\left(\gamma_{J}\right)=E_{J}^{\prime}$ for all $J \in M_{d^{\prime}-1}\left(L_{J}^{\prime}\right.$ is the lattice generated by $\left\{e^{\prime}\right\}_{J}$ ). By $L^{\prime}$-invariance of $T_{0}$, it also contains the $L^{\prime}$-orbit of $E_{J}^{\prime}$. QED 


\section{Relation with Janner-Janssen's Cut Method: The Vertices}

The "cut method" was developped by Janssen [9] and others [10] as an alternative method to build discrete models for quasiperiodic crystals. Its original form was devised to give structural models of modulated crystals [17].

The standard formulation of the cut method is the following: suppose we are given an orthogonal decomposition $\mathbf{R}^{n}=E \oplus E^{\prime}$, where $E$ represents the physical space and where $E^{\prime}=E^{\perp}$. Let $A$ be a regular bounded subset of $E^{\prime}$ (the "atomic surface") and let $\Gamma=\left\{A+\xi \mid \xi \in \mathbf{Z}^{n}\right\}=A+\mathbf{Z}^{n}$ denote the set of lattice translates of $A$. To any $t$ in $E^{\prime}$, there corresponds a structure $X_{t}$ defined as the intersection $(E+t) \cap \Gamma$. The pattern $X_{t}$ is a discrete set of sites since, for any $\xi$ in $\mathbf{Z}^{n},(E+t)$ $\cap(A+\xi)$ is either empty or reduced to a point.

Remark V.1. It can be required that $E+t$ never intersects the boundary $\left\{\partial A+\xi \mid \xi \in \mathbf{Z}^{n}\right\}$ of the atomic surfaces. Such a condition eliminates a set of translations $T_{0}=\bigcup_{x^{\prime} \in L^{\prime}}\left(\partial A+x^{\prime}\right)$ which is of Lebesgue measure zero as long as $A$ is regular in $E^{\prime}$. For the remaining generic translations $t$, the structure $(E+t) \cap \Gamma$ is independent of $A$ being open or closed.

We now proceed to identify the vertices of the quasiperiodic tiling $\mathbb{P}$ with those obtained by an appropriate cut method. by:

For any multiplet $I$ in $\{1, \ldots, n\}$, let $w_{I}$ denote the set of $2^{|I|}$ vertices of $\gamma_{I}$ given

$$
w_{I}=\left\{\sum_{i \in I} \lambda_{i} \varepsilon_{i} \mid \lambda_{i}=0 \text { or } 1\right\}
$$

Define $v_{I}$ and $v_{I}^{\prime}$ as the projections of $w_{I}$ on $E$ and $E^{\prime}$ :

$$
v_{I}=\left\{\sum_{i \in I} \lambda_{i} e_{i} \mid \lambda_{i}=0 \text { or } 1\right\}, \quad v_{I}^{\prime}=\left\{\sum_{i \in I} \lambda_{i} e_{i}^{\prime} \mid \lambda_{i}=0 \text { or } 1\right\}
$$

The sets $\gamma_{I}, D_{I}$, and $D_{I}^{\prime}$ are the convex hulls of $w_{I}, v_{I}$, and $v_{I}^{\prime}$ respectively. If $|I| \leqq d$, $v_{I}$ is the set of vertices of $D_{I}$; similarly, if $|I| \leqq d^{\prime}, v_{I}^{\prime}$ is the set of vertices of $D_{I}^{\prime}$.

Let $t$ be a generic translation and consider the tiling $\mathbb{P}_{t}$ defined in Sect. IV.

For any $d$-multiplet $I$ of $\{1, \ldots, n\}$ and $\xi$ in $\mathbf{Z}^{n}$ such that $E+t$ intersects $\Delta_{I}+\xi$, the tile $D_{I}+x$, where $x=\pi(\xi)$, belongs to the tiling. The corresponding set of vertices of this tile, $v_{I}+x$, is thus given by the intersection of $E+t$ with $\left(v_{I}-D_{I^{c}}^{\prime}\right)+\xi$; actually, for all $s \in v_{I}, s-D_{I^{c}}^{\prime}$ is a subset of the boundary of the cell $\Delta_{I}=D_{I}-D_{I^{c}}^{\prime}$.

In other words (and up to a global translation $-t$ ), the set $X_{t}$ of vertices of the tiling $\mathbb{P}_{t}$ is given by the following intersection (see Definition III.1 and Proposition IV.1):

$$
X_{t}=(E+t) \cap\left\{\bigcup_{I, \xi}\left[\left(v_{I}-D_{I^{c}}^{\prime}\right)+\xi\right]\right\} .
$$

More generally, the set of $q$-facets $(0 \leqq q \leqq d)$ of the quasiperiodic tiling $\mathbb{P}_{t}$ is given by the intersection of $E+t$ with $\bigcup_{I, \xi}\left[\left(F_{I}-D_{I^{c}}^{\prime}\right)+\xi\right]$, where $F_{I}$ denotes the set of $q$-facets of $D_{I}$. 

Lemma V.1. The union $\bigcup_{I . \xi}\left[\left(v_{I}-D_{I^{c}}^{\prime}\right)+\xi\right]$ over all d-multiplets $I$ of $\{1, \ldots, n\}$ and all
$\xi \in \mathbf{Z}^{n}$ is equal to

$$
\Gamma=\left\{-K+\xi \mid \xi \in \mathbf{Z}^{n}\right\},
$$

where $K=\pi^{\prime}\left(\gamma_{n}\right)$ is the orthogonal projection of the unit cube $\gamma_{n}$ on $E^{\prime}$.

Proof. If $\xi=x+x^{\prime}$ belongs to $\mathbf{Z}^{n}$, then $x+\mathbf{Z}^{n}=-x^{\prime}+\mathbf{Z}^{n}$ (this is a trivial identity on the torus). Therefore:

$$
\mathbf{Z}^{n}+\bigcup_{I \in M_{d}}\left(v_{I}-D_{I^{c}}^{\prime}\right)=\bigcup_{I}\left(\mathbf{Z}^{n}+v_{I}-D_{I^{c}}^{\prime}\right)=\bigcup_{I}\left(\mathbf{Z}^{n}-v_{I}^{\prime}-D_{I^{c}}^{\prime}\right)=\mathbf{Z}^{n}-\bigcup_{I}\left(v_{I}^{\prime}+D_{I^{c}}^{\prime}\right) .
$$
Finally, the identity $\bigcup_{I}\left(v_{I}^{\prime}+D_{I^{c}}^{\prime}\right)=K$ follows from $P 4$, proved in the
appendix. QED

This lemma implies that for any generic translation $t$, the set of vertices (5.1) of the quasiperiodic tiling is identical to the set of vertices $(E+t) \cap \Gamma$ corresponding to Janner and Janssen's method.

Next, we show that, for a generic translation $t$, the set of vertices $X_{t}$ of the quasiperiodic tiling is the projection of the set

$$
\Xi_{t}=\left\{\xi \mid \xi \in \mathbf{Z}^{n} \text { and }(E+t) \cap(-K+\xi) \neq \emptyset\right\},
$$

and that the mapping $\Xi_{t} \rightarrow X_{t}$ is one to one.

Consider a general structure $(E+t) \cap \Gamma$ given by the cut method, where $\Gamma=\mathbf{Z}^{n}+A$, and where $A$ is an arbitrary atomic surface. Any vertex $x$ is given by at least one intersection $(E+t) \cap(A+\xi)$ with $x=\pi(\xi)$. However, when the sublattice $\Lambda_{E^{\prime}}=E^{\prime} \cap \mathbf{Z}^{n}$ is not reduced to $\{0\}$, the atomic surface $A+\xi$ associated to $x$ is not necessarily unique; this situation would occur if there existed $\zeta \neq \xi$ such that $A+\xi$ and $A+\zeta$ had the same intersection with $E+t$; in such a case, $\xi-\zeta$ would belong to both $A_{E^{\prime}}$ and $A-A$. The following lemma shows that this is impossible when $A=-\pi^{\prime}\left(\gamma_{n}\right)$.

Lemma V.2. In the settings of Sect. II, let $K^{0}=\pi^{\prime}\left(\gamma_{n}^{0}\right)$ denote the projection of the open unit cube $\gamma_{n}^{0}$ onto $E^{\prime}$. Then $\Lambda_{E^{\prime}} \cap\left(K^{0}-K^{0}\right)=\{0\}$.

Proof. In the standard basis, the components $v_{i}$ of $v=\xi-\zeta=\sum v_{i} \varepsilon_{i}$ are integers. If $v$ belongs to $K^{0}-K^{0}$, there exist reals $\left.\lambda_{i} \in\right]-1,+1\left[\right.$ such that $v=\sum \lambda_{i} e_{i}^{\prime}$ (in the notations of II); set $\lambda=\sum \lambda_{i} \varepsilon_{i}$, then $v=\pi^{\prime}(\lambda)$ so that $v-\lambda$ belongs to $E$. It follows from the orthogonality of $E$ and $E^{\prime}$ that $(v, v-\lambda)=\sum v_{i}\left(v_{i}-\lambda_{i}\right)=0$; since each term satisfies $v_{i}\left(v_{i}-\lambda_{i}\right) \geqq v_{i}^{2}-\left|v_{i}\right| \geqq 0$ we have $v_{i}\left(v_{i}-\lambda_{i}\right)=0$ for all $i$, which implies either $v_{i}=0$ or $v_{i}=\lambda_{i}$; finally $v_{i}=0$ in all cases since $\left|\lambda_{i}\right|<1$. QED

It follows from this lemma that for any generic translation $t$ the set $\Xi_{t}(5.2)$ has a one to one projection on $X_{t}=(E+t) \cap \Gamma$.

\section{The Strip Method}

The "strip" associated to the subspace $E$ and the basis $K \subset E^{\prime}$ is the cylinder $S$ defined by:

$$
S=E+K=E+\gamma_{n}
$$


The strip selects points of $\mathbf{Z}^{n}$ which are then projected onto $E$ to generate the following set (we let $S_{t}=S+t$ ):

$$
X_{t}=\pi\left(S_{t} \cap \mathbf{Z}^{n}\right) .
$$

This set is discrete and quasiperiodic and it coincides with the set obtained through the $\mathbf{J}-\mathbf{J}$ section method. To prove this assertion, note that a point $\xi \in \mathbf{Z}^{n}$ belongs to the strip $S_{t}$ iff $(\xi+E) \cap(K+t) \neq \emptyset$. Rewrite this as $E \cap(\xi-K-t) \neq \emptyset$. Since the last intersection is precisely $\{x=\pi(\xi)\}$ when it is non-empty, we deduce that

$$
X_{t}=E \cap\left(\mathbf{Z}^{n}-K-t\right) .
$$

This expression is identical to the one in Sect. V [Eq. (5.1) and Lemma V.1]. According to what we said in Sect. V, this proves that the set of vertices of the quasiperiodic tiling may, as well, be generated by the strip-projection method.

In this section, we shall show more. Not only the vertices correspond to lattice points, but also the whole tiling is the projection of a continuous faceted surface of the lattice $\mathbf{Z}^{n}$. Moreover this faceted surface is the unique one which is contained in the strip.

The non-degeneracy hypothesis II.8 insures that the tiles $\Delta_{I, \xi}$ of the oblique tiling $\mathbb{O}$ are regular polytopes in $\mathbf{R}^{n}$. The regular sections $\mathbb{P}_{t}(4.5)$ are indexed by [see (4.3-4)]

$$
t \in E^{\prime} \backslash T_{0} .
$$

There are various ways to characterize the tiles of the quasiperiodic tiling. We collect them in a theorem which is followed by a description of the underlying geometry.

Theorem VI.1. Given a $d$-index $I \in M_{d}$ and $\xi \in \mathbf{Z}^{n}$, set $\xi=x+x^{\prime}$ with $x=\pi(\xi)$ and $x^{\prime}=\pi^{\prime}(\xi)$. The following are equivalent:

$1^{\circ} E \cap\left(\Delta_{I, \xi}-t\right) \neq \emptyset$,

$2^{\circ} E \cap\left(\Delta_{I, \xi}-t\right)=x+D_{I}$,

$3^{\circ} E \cap\left(\xi-D_{I^{c}}^{\prime}-t\right) \neq \emptyset$,

$4^{\circ} E \cap\left(\xi-D_{I^{c}}^{\prime}-t\right)=\{x\}$,

$5^{\circ} x^{\prime} \in D_{I^{c}}^{\prime}+t$,

$6^{\circ} \xi \in S_{I}+t$, where $S_{I}=E+D_{I^{c}}^{\prime}$

$7^{\circ} \pi^{\prime}\left(\xi+\gamma_{I}\right) \subset K+t$, where $K=\pi^{\prime}\left(\gamma_{n}\right)$.

$8^{\circ} \xi+\gamma_{I} C S+t$, with $S=E+K$.

Proof. $2^{\circ} \Rightarrow 1^{\circ}$ and $4^{\circ} \Rightarrow 3^{\circ}$ are trivial.

$5^{\circ} \Leftrightarrow 6^{\circ}$ and $7^{\circ} \Leftrightarrow 8^{\circ}$ are direct consequences of the definitions.

For any subset $A \subset \mathbf{R}^{n}$ and any $y \in E$, we have

$$
E \cap(y+A)=(E \cap A)+y .
$$

Apply this identity to $1^{\circ}$ using Definition III.1:

$$
\begin{aligned}
E \cap\left(\Delta_{I, \xi}-t\right) & =\bigcup_{y \in D_{I}}\left(E \cap\left(y-D_{I^{c}}^{\prime}+\xi-t\right)\right) \\
& =\left(E \cap\left(\xi-D_{I^{c}}^{\prime}-t\right)\right)+D_{I} .
\end{aligned}
$$


The right-hand side of $(6.5)$ is non-empty iff $E \cap\left(\xi-D_{I^{c}}^{\prime}-t\right) \neq \emptyset$; this proves $1^{\circ} \Leftrightarrow 3^{\circ}$. Equation (6.5) proves $2^{\circ} \Leftrightarrow 4^{\circ}$ as well.

$3^{\circ} \Leftrightarrow 4^{\circ}$ follows from the general fact $E \cap A \subseteq \pi(A)$.

Project $3^{\circ}$ or $4^{\circ}$ onto $E^{\prime}$. They both lead to

$$
\left(x^{\prime}-D_{I^{c}}^{\prime}-t\right) \cap\{0\} \neq \emptyset \text {, }
$$

which is equivalent to $5^{\circ}$. Thus $3^{\circ} \Leftrightarrow 5^{\circ}$ and $4^{\circ} \Leftrightarrow 5^{\circ}$.

$5^{\circ} \Rightarrow 7^{\circ}$ : By $5^{\circ}, \pi^{\prime}\left(\xi+\gamma_{I}\right)=x^{\prime}+D_{I}^{\prime} \subset D_{I^{c}}^{\prime}+D_{I}^{\prime}+t=\pi^{\prime}\left(\gamma_{I^{c}}+\gamma_{I}\right)+t=\pi^{\prime}\left(\gamma_{n}\right)+t$.

$7^{\circ} \Rightarrow 5^{\circ}: 7^{\circ}$ means that $x^{\prime}-t$ belongs to the " $K$-polar" of $D_{I}^{\prime}$ (which, by convexity, is also the $K$-polar of the set of vertices of $\left.D_{I}^{\prime}\right)$. It is shown in the appendix (P3 and Remark VIII.1) that $K-\operatorname{pol}\left(D_{I}^{\prime}\right)=D_{I^{c}}^{\prime}$. QED

Corollary VI.1. Let $t$ be a regular shift (6.3) and let $Z_{t}$ be the subset of $M_{d} \times \mathbf{Z}^{n}$ consisting of all pairs $(I, \xi)$ satisfying one of the conditions of the theorem. Then the quasiperiodic tiling is given by

$$
\mathbb{P}_{t}=\left\{x+D_{I} \mid x=\pi(\xi),(I, \xi) \in Z_{t}\right\} .
$$

Proof. The proof is simply achieved by writing the definition (4.5) explicitly in terms of Definition III.1 and using statements $1^{\circ}$ and $2^{\circ}$ of the theorem.

Interpretation. Statements $3^{\circ}$ and $4^{\circ}$ are at the origin of the link between the vertices of the tiling and the discrete set generated by a $\mathbf{J}-\mathbf{J}$ section method. Actually, the distinguished $d^{\prime}$-boundary of $\mathbb{O}$ contains the periodic set $\mathbf{Z}^{n}-K$. All the details were presented in Sect. V.

Statements $5^{\circ}$ to $8^{\circ}$ allow to connect the quasiperiodic tiling with the strip or projection method. The cylinder $S$ appearing in $8^{\circ}$ is the strip; the cylinder $S_{I}$ is a substrip of $S$. Statement $6^{\circ}$ means that the set of sites to which are attached the replicates of a prototile $D_{I}$ is the projection $\pi\left(\Xi_{I}\right)$ of the lattice points sitting in the $\operatorname{strip} S_{I}$ :

$$
\Xi_{I}=\mathbf{Z}^{n} \cap\left(S_{I}+t\right)
$$

The union of the sets $\Xi_{I}$ over $M_{d}$ is of course $\Xi_{t}(5.2)$.

Since a necessary and sufficient condition for a tile $D_{I}$ to be present at $x$ in (6.6) is that $x^{\prime}=\pi^{\prime}(\xi)$ satisfies $5^{\circ}$, we name $D_{I^{\circ}}^{\prime}$ the domain of existence of the prototile $D_{I}$. As stated above (6.7), $D_{I^{c}}^{\prime}$ is the basis of the strip generating, through the stripprojection algorithm, the translations involved in the congruence class $\left\{D_{I}+x \in P_{t}\right\}$.

By $2^{\circ}$ of the theorem, every tile $x+D_{I}=\pi\left(\xi+\gamma_{I}\right)$ is the projection of a $d$-facet of the lattice $\mathbf{Z}^{n}$. Since both $D_{I}$ and $\gamma_{I}$ are non-degenerate $d$-dimensional polyhedra, $\pi$ defines a 1-1 linear mapping between $x+D_{I}$ and $\xi+\gamma_{I}$. On the other hand, statement $8^{\circ}$ asserts that the facet $\xi+\gamma_{I}$ lies in the strip $S+t$ so that, if we define the faceted surfaces $F_{I}$ as the unions of all facets $\xi+\gamma_{I}$ involved in the theorem for fixed $I$, and $F$ as $\bigcup_{I} F_{I}$ (thus $F$ is the union of all the facets of $Z^{n}$ with coordinates in $Z_{t}$ of Corollary VI.1), then we are guaranteed that $F \subset S_{t}$. Moreover, by (6.6) and statement $8^{\circ}$ of the theorem, $F$ contains all the $d$-facets of $\mathbf{Z}^{n}$ which are included in $S_{t}$. Therefore $F$ is the unique $d$-dimensional facetted surface selected by $S_{t}$.

It is clear that $F$ is in $1-1$ correspondence with the tiling. We state this in: 
Corollary VI.2. $\left.\pi\right|_{F}: F \rightarrow E$ is a piecewise linear isomorphism.

Proof. That $\left.\pi\right|_{\xi+\gamma_{I}}$ is (the restriction of) a $1-1$ linear mapping from $\xi+\gamma_{I}$ to $x+D_{I}$ has been argued before. That the map $\left.\pi\right|_{F}$ is onto is another way of saying that the tiling (6.6) covers $E$. The injective nature of $\left.\pi\right|_{F}$ is related to the uniqueness of the facet $\xi+\gamma_{I}$ above $x+D_{I}$. This uniqueness is obvious in $2^{\circ}$ of theorem, provided the section is regular (6.3) and (4.3). QED

Remarks VI.1. a) When the orientation of $E^{\prime}$ is irrational, $\pi^{-1}(0) \cap \mathbf{Z}^{n}=\{0\}$, a tile $x+D_{I}$ is the projection of a unique facet $\xi+\gamma_{I}$; moreover, the uniqueness holds throughout the whole lattice $\mathbf{Z}^{n}$. Indeed, if $\zeta_{1}$ and $\zeta_{2}$ are two points of $\mathbf{Z}^{n}$ projecting onto the same vertex $z$ of $x+D_{I}$, then $\pi\left(\zeta_{1}-\zeta_{2}\right)=0$ which, by the irrationality condition, implies $\zeta_{1}=\zeta_{2}$. The uniqueness for the vertices implies the uniqueness of the facet.

b) When, on the other hand, there is a nontrivial intersection lattice $\Lambda_{E^{\prime}}$ (see II.7), if $x+D_{I}=\pi\left(\xi+\gamma_{I}\right)$ for some facet of $\mathbf{Z}^{n}$, then any facet of the orbit $\Lambda_{E^{\prime}}+\left(\xi+\gamma_{I}\right)$ projects onto the same tile $x+D_{I}$. Thus, in this case, the uniqueness only holds within the strip $S_{t}$, in the sense that there is one and only one facet in $S_{t} \cap\left(\Lambda_{E^{\prime}}+\xi+\gamma_{I}\right)$ whose projection is $x+D_{I}$; the uniqueness within $S_{t}$ may be proved by the argument presented in Remark a) using, moreover, Lemma V.2.

From the tiling of $E$, we may infer another property for the faceted surface to which it is isomorphic, namely that $F$ is a simply connected $d$-dimensional manifold without boundary.

Corollary VI.3. Let $s$ be the section ${ }^{7} s=\left(\left.\pi\right|_{F}\right)^{-1}$. Then $s$ is a continuous map $E \rightarrow \mathbf{R}^{n}$.

Proof. Since $s$ is linear inside every tile of $P_{t}$, all we have to check is continuity across the vertices. Let $x$ be a vertex of $P_{t}$. Suppose that $\xi$ and $\zeta$ are two limit points of $s(x)$. By definition, $\xi$ and $\zeta \in \mathbf{Z}^{n}$ and, by $8^{\circ}$ of Theorem VI.1, both $\xi$ and $\zeta$ belong to $S+t$. This implies in particular that $\pi^{\prime}(\xi-\zeta) \in K-K$. Now $\pi(\xi)-\pi(\zeta)=0$ means that $\xi-\zeta \in \Lambda_{E^{\prime}}$. Therefore, by Lemma V.2 and Remark V.1, $\xi-\zeta=0$. QED

\section{Conclusions}

We conclude here by two comments on a more general level in the geometry of tilings. The first one is related to tilings involving the same tiles as the quasiperiodic ones discussed in the course of the present article but possibly different long range order; the second one deals with tilings made of different tiles but characterized by the same type of quasiperiodic ordering.

Among the basic open questions related to quasiperiodic tilings is the following: can the global (long range) quasiperiodic ordering be inferred from local criteria such as the number and shape of the prototiles, matching rules, etc. For the famous 2-dimensional Penrose tilings, a positive answer was given by de Bruijn in [7]. There, the local prescriptions consist e.g. in drawing arrows on the sides of the rhombi and fulfilling a simple set of matching rules; any covering of the plane by Penrose's rhombi satisfying the rules is quasiperiodic. Another type of local

${ }^{7}$ Section is meant here in the topological sense as an inverse of the projection 
prescriptions, albeit related to the previous ones, is to select a restricted number of incidences among all those which are possible a priori (an incidence around a vertex $x$ of the tiling is the pattern of all the tiles sharing $x$ as a common vertex).

In this direction, we claim the following: in any tiling built from a "compatible" non-planar section through the oblique one, the list of incidences is the same as in the quasiperiodic case. What we mean by compatible is the following - the claim is proved in the argument below:

A section over $E$ is a continuous map $s: E \rightarrow \mathbf{R}^{n}$ which maps diffeomorphically $E$ onto $\sigma=s(E)$. Moreover, a section is compatible with tiling if it satisfies

$$
\sigma \cap \partial^{(E)} \mathbb{D}=\emptyset,
$$

where $\partial^{(E)} \mathbb{D}$ is given in (4.2). A compatible section (simply: $c$-section) crosses the oblique tiling $\mathbb{Q}$ and thereby selects a simply connected chain of oblique tiles in $\mathbf{R}^{n}$; by projection, such a chain defines a unique tiling of $E$ :

$$
\mathbb{T}_{s}=\pi(\sigma \cap \mathbb{D}) .
$$

The prototiles are the same as those of the strictly quasiperiodic ones which were obtained, in Sect. IV, by letting $s$ be $s(x)=x+t$ for suitable $t \in E^{\prime}$; but the long range order may differ from being quasiperiodic when $\sigma$ is not an affine subspace. Non-planar sections over $E$ may conveniently describe "perturbed" quasiperiodic tilings, defects in the ordering, phasons etc., they may, as well, be considered in trying to understand why and how the quasiperiodicity sets in among the various possible orderings compatible with a prescribed set of prototiles or, more generally, local constraints. The above claim means that compatible "undulations" of the section keep constant the protoset and the incident list. Whether such a list implies quasiperiodicity, i.e. whether compatible sections are necessary planar (or homotopic to planar ones) is not yet known.

Argument. To prove the claim, note that the vertices are given by $\pi(\sigma \cap \Gamma)$, where $\Gamma=\mathbf{Z}^{n}-K$. Suppose that $x$ is such a vertex. This means that $\sigma$ cuts $\xi-K$ for some $\xi \in \mathbf{Z}^{n} \cap \pi^{-1}(x)$; we let $y$ be this intersection point. On the other hand, there is an affine subspace $E+t$ containing $y$, so that the induced quasiperiodic tiling $\mathbb{P}_{t}$ (see Proposition IV.1) shares, with $\mathbb{T}_{s}$ given by (7.2), the same vertex $x$. Now, by continuity, the incidences around $x$ in $\mathbb{P}_{t}$ and in $\mathbb{T}_{s}$ coincide since, in both cases, the incidence is the projection of all the oblique tiles having $y$ in their boundary [notice that $y$ necessarily lies in the open subset $(\xi-K) \backslash \hat{\partial}^{(E)} \mathbb{D}$ of $\xi-K$ by hypothesis (7.1)].

Back to quasiperiodicity, a direct characterization of such an ordering for tilings without explicitly referring to particular point symmetries or to Fourier transforms (the Fourier transform of the quasiperiodic tiling is not a measure but a wilder distribution) consists in viewing quasiperiodic tilings as affine sections of periodic tilings in higher dimensions. Given a generic affine cut $E$ through a lattice $\Lambda$ imbedded in $\mathbf{R}^{n}$, only a special class of periodic $n$-dimensional tilings are able to produce true tilings of $E$, namely those of the "oblique" type: a tiling is oblique if all its (proto-)tiles are direct sums of a polyhedron in $E$ and a polyhedron in the complementary space $E^{\prime}$. In this paper, we have shown how to build such a tiling starting from $\mathbf{Z}^{n}$ and its related standard fundamental cell, the unit cube $\gamma_{n}$. The corresponding sections - the "canonical" quasiperiodic tilings - have been 
analysed in detail; the canonical tiles are parallelohedra obtained as projected facets of the lattice; moreover we have proved that the whole canonical quasiperiodic tiling itself is the projection of a simply connected faceted surface [of dimension $d=\operatorname{dim}(E)]$ which is the unique faceted surface selected the strip $E+\gamma_{n}$.

The canonical tilings, albeit representative, are, of course, not the only ones showing quasiperiodic order. A number of different tilings with different prototiles and local patterns could be conceived. The long range order is related to the imbedding lattice $\Lambda$ and to the orientation of the subspace $E$ with respect to it, as it clearly appears, e.g., in the Fourier transforms. However, it is not difficult to build quasiperiodic tilings showing the same type of (pseudo-)symmetries as a given canonical one but which do not correspond to decorating, dissecting, or redistributing the canonical tiles according to a finite number of prescriptions (modulo translations).

Given an arbitrary quasiperiodic tiling, to find a higher dimensional periodic tiling producing the given one by section may be a delicate problem: the polyhedra of $E$ entering the definition of the rectangular tiles are, of course, the prototiles of the quasiperiodic one; on the other hand, as shown in the canonical example, the polyhedra in $E^{\prime}$ would rather be related to the sets of translations involved in the congruence classes of the tiles.

However, the cut method provides a general method to build quasiperiodic tilings and models. It insures, ipso facto, that one gets 1: a true tiling, 2: a quasiperiodic tiling if the section is affine.

\section{Appendix: Zonohedra}

Let $E$ be a $d$-dimensional real vector space and assume that $a_{1}, a_{2}, \ldots, a_{n}$ are $n \geqq d$ vectors spanning $E$.

Definition VIII.1. The zonohedron $Z=Z\left(a_{1}, a_{2}, \ldots, a_{n}\right)$ is the set of $x=\lambda_{1} a_{1}$ $+\lambda_{2} a_{2}+\ldots+\lambda_{n} a_{n}$, where $0 \leqq \lambda_{i} \leqq 1$ for $i=1, \ldots, n$.

Let $V=V\left(a_{1}, a_{2}, \ldots, a_{n}\right)$ denote the set of $2^{n}$ points $x=\lambda_{1} a_{1}+\lambda_{2} a_{2}+\ldots+\lambda_{n} a_{n}$ with $\lambda_{l}=0$ or 1 . $V$ contains the vertices (extremal points) of $Z$ and possibly other interior points if $n>d$.

For any subset $I \subset\{1, \ldots, n\}$ define $Z_{I}=\left\{\sum \lambda_{i} a_{i} \mid \lambda_{i} \in[0,1]\right.$ for $i \in I$ and $\lambda_{i}=0$ for $i \notin I\}$ and let $V_{I}$ denote the corresponding set of $2^{|I|}$ ponts including the vertices of $Z_{l}$.

For any $I C\{1, \ldots, n\}$ and any point $x$ of $Z_{I}$ there exists a system of positive coordinates $\left\{\mu_{\sigma}\right\}_{\sigma \in V_{I}}$, the sum of which equals one, such that $x=\sum_{\sigma \in V_{I}} \mu_{\sigma} \sigma$.

Example 1. If $\varepsilon_{1}, \varepsilon_{2}, \ldots, \varepsilon_{n}$ is the standard basis of $\mathbf{R}^{n}, Z\left(\varepsilon_{1}, \varepsilon_{2}, \ldots, \varepsilon_{n}\right)$ is the unit hypercube of $\mathbf{R}^{n}$.

Example 2. If $\pm e_{1}, \pm e_{2}, \ldots, \pm e_{6}$ are the vertices of a regular icosahedron in $\mathbf{R}^{3}$, $Z\left(e_{1}, e_{2}, \ldots, e_{6}\right)$ is a triacontahedron, a convex polyhedron with 32 (external) vertices, 60 edges and 30 identical facets.

Zonohedra satisfy the following properties:

$P 1$. For any $I \subset\{1, \ldots, n\}, Z_{I}$ is a closed bounded convex subset of $E$. 
$P 2$. For any $I \subset\{1, \ldots, n\}$ the following holds:

$$
\bigcap_{x \in Z_{I}}(Z-x)=\bigcap_{\sigma \in V_{I}}(Z-\sigma) \text {. }
$$

Proof. The inclusion $\bigcap_{x \in Z_{I}}(Z-x) \subset \bigcap_{\sigma \in V_{I}}(Z-\sigma)$ is obvious.

Suppose $I=\left\{i_{1}, \ldots, i_{k}\right\}$ and let $a$ be any point of $\bigcap_{\sigma \in V_{I}}(Z-\sigma)$. For any $\sigma \in V_{I}, a$ belongs to $Z-\sigma$ so that there exist $\lambda_{\sigma, 1}, \ldots, \lambda_{\sigma, n}$ in $[0,1]$ such that $a=\lambda_{\sigma, 1} a_{1}+\ldots+\lambda_{\sigma, n} a_{n}-\sigma$; if $x$ is any point of $Z_{I}$, there exist positive coordinates $\left\{\mu_{\sigma}\right\}_{\sigma \in V_{I}}$ with sum equal to 1 such that $x=\sum \mu_{\sigma} \sigma$; therefore

$a=\left(\sum \mu_{\sigma}\right) a=\left(\sum \mu_{\sigma} \lambda_{\sigma, 1}\right) a_{1}+\ldots+\left(\sum \mu_{\sigma} \lambda_{\sigma, n}\right) a_{n}-x$, thus $a \in Z-x$ and finally

$$
a \in \bigcap_{x \in Z_{I}}(Z-x) \text {. }
$$

P3. For any $I \subset\{1, \ldots, n\}$ the following holds:

$$
\bigcap_{\sigma \in V_{I}}(Z-\sigma)=Z_{I^{c}}, \text { where } I^{c}=\{1, \ldots, n\} \backslash I .
$$

Proof. The inclusion $\bigcap_{\sigma \in V_{I}}(Z-\sigma) \supset Z_{I^{c}}$ is obvious. Let us show that
$(Z-\sigma) \subset Z_{I^{c}}$. $\bigcap_{\sigma \in V^{I}}(Z-\sigma) \subset Z_{I^{c}}$

Assume $I=\{i\}$ and let $x$ be any point of $Z \cap\left(Z-a_{i}\right)$ : there exist $\lambda_{1}, \ldots, \lambda_{n}$ and $\mu_{1}, \ldots, \mu_{n}$ in $[0,1]$ such that $x=\sum \lambda_{j} a_{j}=\sum \mu_{j} a_{j}-a_{i}$; let $\alpha \in[0,1]$ be such that $\alpha \lambda_{i} P 4$. $+(1-\alpha)\left(\mu_{i}-1\right)=0$; then

$x=\alpha x+(1-\alpha) x=\sum_{j \neq i}\left[\alpha \lambda_{j}+(1-\alpha) \mu_{j}\right] a_{j}$ belongs to $Z_{I^{c}}$.

Suppose now that $I=\{i\} \cup J$ and assume that the property holds for $J$. Since $V_{I}=V_{J} \cup\left[V_{J}+a_{i}\right]$,

$$
\bigcap_{\sigma \in V_{I}}(Z-\sigma)=\left[\bigcap_{\sigma \in V_{J}}(Z-\sigma)\right] \cap\left[\bigcap_{\sigma \in V_{J}+a_{v}}(Z-\sigma)\right]=Z_{J^{c}} \cap\left[Z_{J^{c}}-a_{i}\right]=Z_{I^{c}} .
$$

Remark VIII.1. Let $K$ be a subset of $E$. For any $B \subset E$, define the $K$-polar of $B$ as $K-\operatorname{pol}(B)=\{t \in E \mid B+t \subset K\}[13]$. By the simple identity $K$-pol $(B)=\bigcap_{b \in B}(K-b), P 2$ means that $Z$ - $\operatorname{pol}\left(Z_{I}\right)=Z-\operatorname{pol}\left(V_{I}\right)$ and $P 3$ asserts that this polar is $Z_{I^{c}}$.

P4. $Z=Z\left(a_{1}, a_{2}, \ldots, a_{n}\right)$ can be viewed as the union of $\left(\begin{array}{l}d \\ n\end{array}\right) d$-dimensional zonohedra $Z_{I}$, conveniently translated by vectors of $V$, where $I=\left\{i_{1}, \ldots, i_{d}\right\}$ runs over $M_{d}$; these zonohedra have non-overlapping interiors (this partition of $Z$ is not unique if $n>d$ ).

The boundary $\partial Z\left(a_{1}, a_{2}, \ldots, a_{n}\right)$ consists in $2 \cdot\left(\begin{array}{c}d-1 \\ n\end{array}\right)(d-1)$-facets corresponding to opposite pairs of $\left(\begin{array}{c}d-1 \\ n\end{array}\right)(d-1)$-dimensional zonohedra $Z\left(a_{i_{1}}, \ldots, a_{i_{d-1}}\right)$, where $\left\{i_{1}, \ldots, i_{d-1}\right\}$ runs over all subsets of $d-1$ indices in $\{1, \ldots, n\}$.

Proof. The property is obvious for $n=d$ for $Z$ is an hypercube. Let

$$
Z\left(a_{1}, \ldots, a_{n}\right)=Z\left(a_{1}, \ldots, a_{n-1}\right)+Z\left(a_{n}\right)
$$

and assume the property holds for $n-1 ; Z\left(a_{1}, \ldots, a_{n-1}\right)$ is tiled by $\left(\begin{array}{c}d \\ n-1\end{array}\right)$ translated zonohedra with indices in $\{1, \ldots, n-1\}$ and translations in 
$V\left(a_{1}, \ldots, a_{n-1}\right)$. The boundary $\partial Z\left(a_{1}, \ldots, a_{n-1}\right)$ of $Z\left(a_{1}, \ldots, a_{n-1}\right)$ consists in $2 \cdot\left(\begin{array}{l}d-1 \\ n-1\end{array}\right)(d-1)$-facets like $Z\left(a_{i_{1}}, \ldots, a_{i_{d-1}}\right)$, each one occurring in two opposite positions. The translation of $Z\left(a_{1}, \ldots, a_{n-1}\right)$ by $Z\left(a_{n}\right)$ results in adding to $Z\left(a_{1}, \ldots, a_{n-1}\right)$ a "layer" of $\left(\begin{array}{l}d-1 \\ n-1\end{array}\right)$ zonohedra like $Z\left(a_{i_{1}}, \ldots, a_{i_{d-1}}, a_{n}\right)$ $=Z\left(a_{i_{1}}, \ldots, a_{i_{d-1}}\right)+Z\left(a_{n}\right)$, which achieves the proof since $\left(\begin{array}{l}d-1 \\ n-1\end{array}\right)+\left(\begin{array}{c}d \\ n-1\end{array}\right)=\left(\begin{array}{l}d \\ n\end{array}\right)$.

Acknowledgements. We owe to Pierre Collet our being aware of [4]; it is a pleasure to thank him for discussions. One of us, C.O., thanks the Fonds National Suisse for financial support.

\section{References}

1. Adler, R.: Similarity of automorphisms of the torus. Mem. Am. Math. Soc. 98 (1970)

2. Bowen, R.: Ergodic states and the ergodic theory of Anosov diffeomorphisms. Lecture Notes in Mathematics, Vol. 470. Berlin, Heidelberg, New York: Springer 1975

3. Bowen, R.: Proc. Am. Math. Soc. 71, 130-132 (1978)

4. Bedford, T.: Ergodic Theory Dyn. Syst. 6, 325-333 (1985)

5. Shechtman, D., Blech, I., Gratias, D., Cahn, J.W.: Phys. Rev. Lett. 53, 1951 (1984)

6. Les Houches, Workshop on Aperiodic Crystals, 11-22 March 1986; Michel, L., Gratias, D. (eds.). J. Phys. Colloque

7. de Bruijn, N.G.: Kon. J. Nederl. Akad. Wetensch. Proc. A 84, 38-66 (1981)

8. Levine, D., Steinhardt, P.: Phys. Rev. B 34, 596 (1986)

Socolar, J., Steinhardt, P., Levine, D.: Phys. Rev. B 32, 5547 (1985)

9. Janssen, T.: Acta Crystallogr. A 42, 261 (1986)

10. Bak, P.: Phys. Rev. Lett. 56, 861 (1986)

Frenkel, D., Henley, C., Siggia, E.: Phys. Rev. B 34, 3649 (1986)

Socolar, J., Lubensky, T., Steinhardt, P.: Phys. Rev. B 34, 3345 (1986)

11. Kalugin, P.A., Kitaev, A.Yu., Levitov, L.C.: JETP Lett. 41, 145 (1985); J. Physique Lett. 46, L-601 (1985).

Elser, V.: Phys. Rev. Lett. 54, 1730 (1985): Phys. Rev. B 32, 4982 (1985)

Duneau, M., Katz, A.: Phys. Rev. Lett. 54, 2477 (1985)

12. Gähler, F., Rhyner, J.: J. Phys. A 19, 267 (1986)

13. Katz, A., Duneau, M.: J. Physique 47, 181 (1986)

14. de Bruijn, N.G.: Les Houches, Workshop on Aperiodic Crystals, 11-22 March 1986; Michel, L., Gratias, D. (eds.) (J. Phys. Colloque), p. c3-9

15. Bohr, H.: Fastperiodische Funktionen. Berlin, 1932; and Almost periodic functions. New York: Chelsea Co. 1951

16. Besicovitch, A.S.: Almost periodic functions. Cambridge: Cambridge University Press 1932

17. de Wolf, P.M., Janssen, T., Janner, A.: Acta Crystallogr. A 37, 625 (1981)

18. Kramer, P.: Mod. Phys. Lett. B 1, 7-18 (1987) and Int. Mod. Phys. B 1, 145-165 (1987) (where the imbedding dimension is respectively $n=2$ and $n=3$ ). Space-group theory for a nonperiodic icosahedral quasilattice, to be published. In: J. Math. Phys. (in this last work, the proof relies on the particular point symmetry)

19. Penrose, R.: Math. Intell. 2, 32-37 (1979)

20. Rokhsar, D.S., Mermin, N.D., Wright, D.C.: Rudimentary quasicrystallography: the cosahedral and the decagonal reciprocal lattices. Preprint Nov. 1986

21. Cartier, P.: C.R. Acad. Sci. Paris 304, II, 798 (1987)

Communicated by A. Jaffe

Received December 17, 1987 